\title{
Production of free monounsaturated fatty acids by metabolically engineered Escherichia coli
}

\author{
Yujin $\mathrm{CaO}^{\dagger}$, Wei Liu ${ }^{\dagger}$, Xin Xu, Haibo Zhang, Jiming Wang and Mo Xian ${ }^{*}$
}

\begin{abstract}
Background: Monounsaturated fatty acids (MUFAs) are the best components for biodiesel when considering the low temperature fluidity and oxidative stability. However, biodiesel derived from vegetable oils or microbial lipids always consists of significant amounts of polyunsaturated and saturated fatty acids (SFAs) alkyl esters, which hampers its practical applications. Therefore, the fatty acid composition should be modified to increase MUFA contents as well as enhancing oil and lipid production.

Results: The model microorganism Escherichia coli was engineered to produce free MUFAs. The fatty acyl-ACP thioesterase (AtFatA) and fatty acid desaturase (SSI2) from Arabidopsis thaliana were heterologously expressed in E. coli BL21 star(DE3) to specifically release free unsaturated fatty acids (UFAs) and convert SFAs to UFAs. In addition, the endogenous fadD gene (encoding acyl-CoA synthetase) was disrupted to block fatty acid catabolism while the native acetyl-CoA carboxylase (ACCase) was overexpressed to increase the malonyl coenzyme A (malonyl-CoA) pool and boost fatty acid biosynthesis. The finally engineered strain BL21 $\triangle$ fadD/pE-AtFatAssiz\&pA-acc produced $82.6 \mathrm{mg} / \mathrm{L}$ free fatty acids (FFAs) under shake-flask conditions and FFAs yield on glucose reached about 3.3\% of the theoretical yield. Two types of MUFAs, palmitoleate (16:1 $\Delta 9)$ and cis-vaccenate $(18: 1 \Delta 11)$ made up more than $75 \%$ of the FFA profiles. Fed-batch fermentation of this strain further enhanced FFAs production to a titer of $1.27 \mathrm{~g} / \mathrm{L}$ without affecting fatty acid compositions.

Conclusions: This study demonstrated the possibility to regulate fatty acid composition by using metabolic engineering approaches. FFAs produced by the recombinant E. coli strain consisted of high-level MUFAs and biodiesel manufactured from these fatty acids would be more suitable for current diesel engines.

Keywords: Free monounsaturated fatty acids, Thioesterase, Fatty acid desaturase, acyl-CoA synthetase, acetyl-CoA carboxylase
\end{abstract}

\section{Background}

Biodiesel is a mixture of fatty-acid alkyl esters obtained by the transesterification of triglycerides (in most cases, vegetable oils and animal fats) with methanol or ethanol. It is a renewable alternative with the potential to replace the petroleum-based diesel. The properties of a biodiesel fuel including cetane number, density, viscosity, flash point, oxidative stability and cold-filter plugging point, are determined by the structure of its component alcohols and fatty acids [1], whereas the properties of an individual fatty acid depend on the chain length, the occurrence of double bonds and branch chains [2]. For

\footnotetext{
* Correspondence: xianmo@qibebt.ac.cn

${ }^{\dagger}$ Equal contributors

CAS Key Laboratory of Bio-based Materials, Qingdao Institute of Bioenergy and Bioprocess Technology, Chinese Academy of Sciences, Qingdao, China
}

instance, the calorific value of biodiesel increases along with fatty-acid chain length, but the low temperature fluidity decreases with chain length. The longer the chain, the greater the viscosity, and cis double bonds also lower the viscosity [3]. According to a report from the US Department of Energy, the perfect biodiesel would be made only from monounsaturated fatty acids (MUFAs) [4]. An ideal biodiesel composition should have fewer polyunsaturated and saturated fatty acids (SFAs) [5]. High levels of polyunsaturated fatty acids (PUFAs) would negatively impact the oxidative stability and increase nitrogen oxide exhaust emissions, which do not suit diesel engines [6,7]. On the contrary, biodiesel derived from SFAs would have good oxidative stability, but poor fuel properties at low temperatures, which is a disadvantage in winter operation [8]. 
At present, commercially available biodiesel is mostly produced from vegetable oils. Unfortunately, fatty acid composition varies greatly between different vegetable oils. As shown in Table 1, many of these vegetable oils contain a high level of linoleate $(18: 2 \Delta 9,12)$ whereas others contain high amounts of palmitate (16:0). Therefore, the properties of vegetable oil-based biodiesel always need to be adjusted by certain additives to enhance its utilization potential. In addition, the excessive consumption of vegetable oils is a threat to global food security. In recent years, the use of microbial lipids, which can be obtained by fermenting non-food feedstocks, to synthesize biodiesel is attracting increasing attention. A number of oleaginous microorganisms such as microalgae [9], yeasts [10] and fungi [11], have been tested for their capability for lipid accumulation. As a reference microorganism, Escherichia coli possesses many advantages over other microorganisms, for example, a clear genetic background, the convenience of its ability to be genetically modified, and good growth properties with low nutrient requirements $[12,13]$. It has been widely studied for production of fatty acids $[14,15]$, or directly biodiesel $[16,17]$, and shows a promising prospect for industrial application. Previous studies have demonstrated that thioesterase expression is a key step to produce free fatty acids (FFAs) in E. coli. Heterologous expression of different thioesterases would lead to accumulation of significant amounts of FFAs [18]. Blocking fatty acid consumption is also critical for FFA production in E. coli. A promising alternative route to produce FFAs would involve reversing $\beta$-oxidation so that it operates anabolically to the fatty-acid biosynthesis direction [19]. The irreversible synthesis of malonyl-CoA catalyzed by acetyl-CoA carboxylase is another key regulatory point for fatty acid production. Overexpression of this enzyme could increase the final titer of FFAs in the engineered strain [20]. Compared with vegetable oils and microalgal lipids, biodiesel manufactured by $E$. coli does not contain PUFAs. However, SFAs make up the majority of $E$. coli lipids [21]. Modifying the fatty acid compositions of $E$. coli is critical to promote its practical application in the biodiesel industry.

In this study, four distinct genetic alterations targeted at free MUFA production were introduced into the host strain E. coli BL21 star(DE3) (Figure 1), including heterologous alterations expressing a fatty acyl carrier protein (ACP) thioesterase to render $E$. coli capable of producing FFAs; introducing a fatty-acid desaturase to convert SFAs to unsaturated fatty acids (UFAs); knockout of the endogenous $f a d D$ gene, which encodes the acyl-CoA synthetase, to block native fatty-acid catabolism; and further overexpression of the acetyl-CoA carboxylase to increase the malonyl-CoA pool and boost fatty-acid biosynthesis. The finally engineered strain was cultured under fed-batch conditions to evaluate its ability to produce MUFAs.

\section{Results and discussion}

\section{Production of free unsaturated fatty acids by a specific thioesterase}

As a model organism to study fatty-acid biosynthesis, $E$. coli has a type II FAS (FAS II) system, which is found in most bacteria and plants [27]. In this biosynthetic system, fatty acids are bound to ACP and catalyzed by a series of discrete, mono-functional enzymes [28]. However, the fatty acids in E. coli are only used to synthesize membrane phospholipids and lipid A. Few FFAs are produced by wild-type E. coli strains. Fatty acid thioesterase (Fat) is an enzyme that cleaves the fatty acid thioester bond to coenzyme A (CoA) or ACP. The E. coli genome only carries two acyl-CoA thioesterases (tes $A$ and tesB), which catalyze the hydrolytic cleavage of fatty acyl-CoA thioesters and function in the process of fatty-acid degradation [29]. Although these two enzymes can also cleave the bonds of fatty acyl-ACP thioesters, the catalytic efficiency is much lower than acyl-CoA esters of the same length [30].

Acyl-ACP thioesterases can hydrolyze the thioester bond between the acyl moiety and ACP. These enzymes play an essential role in chain termination during de novo fatty-acid synthesis in higher plants [31]. Expression of plant acyl-ACP thioesterases can result in dramatic changes in the fatty-acid profiles both in E. coli cell membrane and culture supernatant [32-34]. According to the substrate specificity, there are two types of acyl-ACP thioesterases, FatA and FatB. Substrate specificity of these isoforms depends on the chain length and

Table 1 Fatty acid compositions of several typical vegetable oils

\begin{tabular}{lcccccccccc}
\hline Vegetable oils & $\mathbf{1 2 : 0}$ & $\mathbf{1 4 : 0}$ & $\mathbf{1 6 : 0}$ & $\mathbf{1 6 : 1}$ & $\mathbf{1 8 : 0}$ & $\mathbf{1 8 : 1}$ & $\mathbf{1 8 : 2}$ & $\mathbf{1 8 : 3}$ & $\mathbf{2 2 : 1}$ & References \\
\hline Soybean oil & NR & NR & $11 \%$ & NR & $4 \%$ & $23.4 \%$ & $53.2 \%$ & $7.8 \%$ & Traces & {$[22]$} \\
Corn oil & NR & NR & $10.3 \%$ & NR & $1.0 \%$ & $30.3 \%$ & $58.0 \%$ & $0.4 \%$ & NR & {$[23]$} \\
Rapeseed oil & NR & NR & $5.51 \%$ & NR & $2.17 \%$ & $58.33 \%$ & $19.89 \%$ & $9.13 \%$ & $4.59 \%$ & {$[24]$} \\
Palm oil & $0.1 \%$ & $1.0 \%$ & $42.8 \%$ & NR & $4.5 \%$ & $40.5 \%$ & $10.1 \%$ & $0.2 \%$ & NR & {$[25]$} \\
Jatropha oil & NR & NR & $15.32 \%$ & $1.33 \%$ & $4.06 \%$ & $35.38 \%$ & $43.34 \%$ & NR & NR & {$[26]$} \\
\hline
\end{tabular}




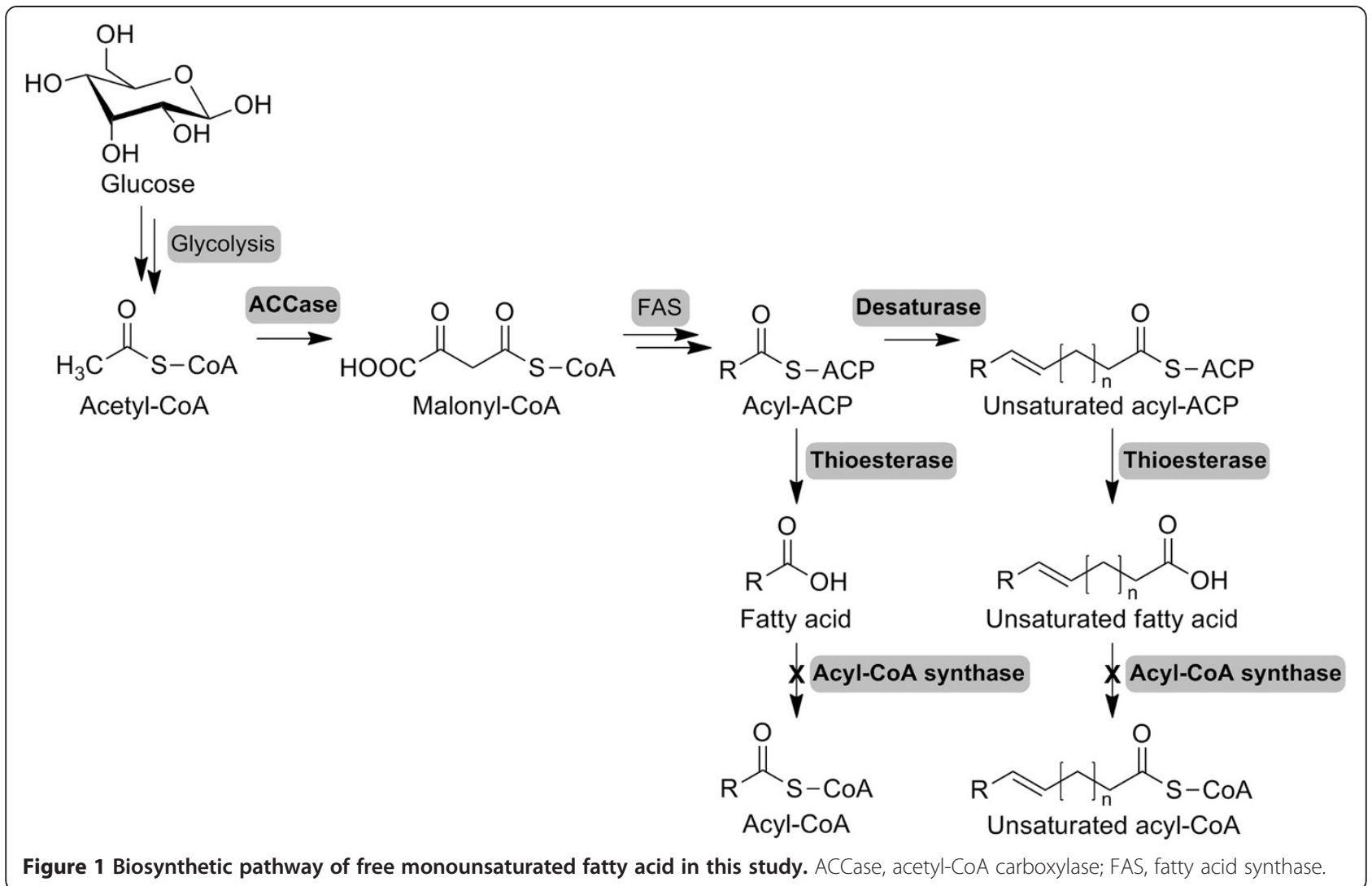

saturation level of fatty acids. The highest activity of FatA is with unsaturated acyl-ACP whereas FatB prefers palmitoyl (16:0)-ACP as its optimal substrate [35]. The Arabidopsis thaliana AtFatA gene encodes a thioesterase with preference towards oleoyl (18:1 $\Delta 9)$-ACP. The catalytic efficiencies of AtFatA are always several-fold higher for the monoenes than their saturated counterparts [36].

In order to produce free UFAs, the thioesterase AtFatA was heterologously expressed in E. coli BL21 star(DE3). The coding region of this gene was cloned into the pACYCduet-1 expression vector under T7 promoter, resulting pA-AtFatA. The recombinant constructs were checked by restriction enzyme digestion and DNA sequencing. To verify the expression level of the recombinant protein, E. coli competent cells were transformed by the expression vector pA-AtFatA and grown in liquid LB medium to an optical density $(\mathrm{OD})_{600}$ of 0.6 followed by induction using $0.1 \mathrm{mM}$ isopropyl- $\beta$-D-thiogalactopyranoside (IPTG). Figure 2 showed the gel electrophoresis patterns of samples from different recombinant strains analyzed with coomassie brilliant blue staining (to visualize all proteins). Lane 2 was the cell lysates of strain BL21/pA-AtFatA. It could be found that AtFatA was soluble expressed in $E$. coli (corresponding to the band of molecular weight $40.8 \mathrm{kDa})$.
To evaluate the effects of AtFatA on FFA production, the recombinant strain BL21/pA-AtFatA was cultured using M9 minimal medium under shake-flask conditions. After being induced for $16 \mathrm{~h}$, FFAs were extracted, derivatized and identified by gas chromatography-mass spectrometry (GC-MS) analysis. As shown in Figure 3, three types of fatty acid, that is, palmitoleate $(16: 1 \Delta 9)$, palmitate $(16: 0)$ and cis-vaccenate $(18: 1 \Delta 11)$ made up the major components of the AtFatA-expressing strain. In addition, trace amounts of myristate, cis-methylene-9,10-hexadecanoate and cis-methylene-11,12-octadecanoate were also detected. The two types of cyclopropane fatty acids (CFAs) were formed by modification of the double bond of UFAs in the phospholipids, which was catalyzed by CFA synthase $[37,38]$. CFAs should only exist in the cellular membrane and the appearance of CFAs might be due to the fact that some of them were extracted along with FFAs by ethyl acetate. To determine the contents of these fatty acids, the FFA profiles were further analyzed by gas chromatography (GC) coupled with a flame ionization detector (FID). The amount of each fatty acid was computed from the area under the corresponding peak. The recombinant strain carrying pA-AtFatA overproduced 16:1 and 18:1 fatty acid. Free palmitoleate and cis-vaccenate levels of this strain were $45.6 \%$ and $21.5 \%$, respectively, and the ratio of UFA to SFA reached 2:1. The total FFA production was 


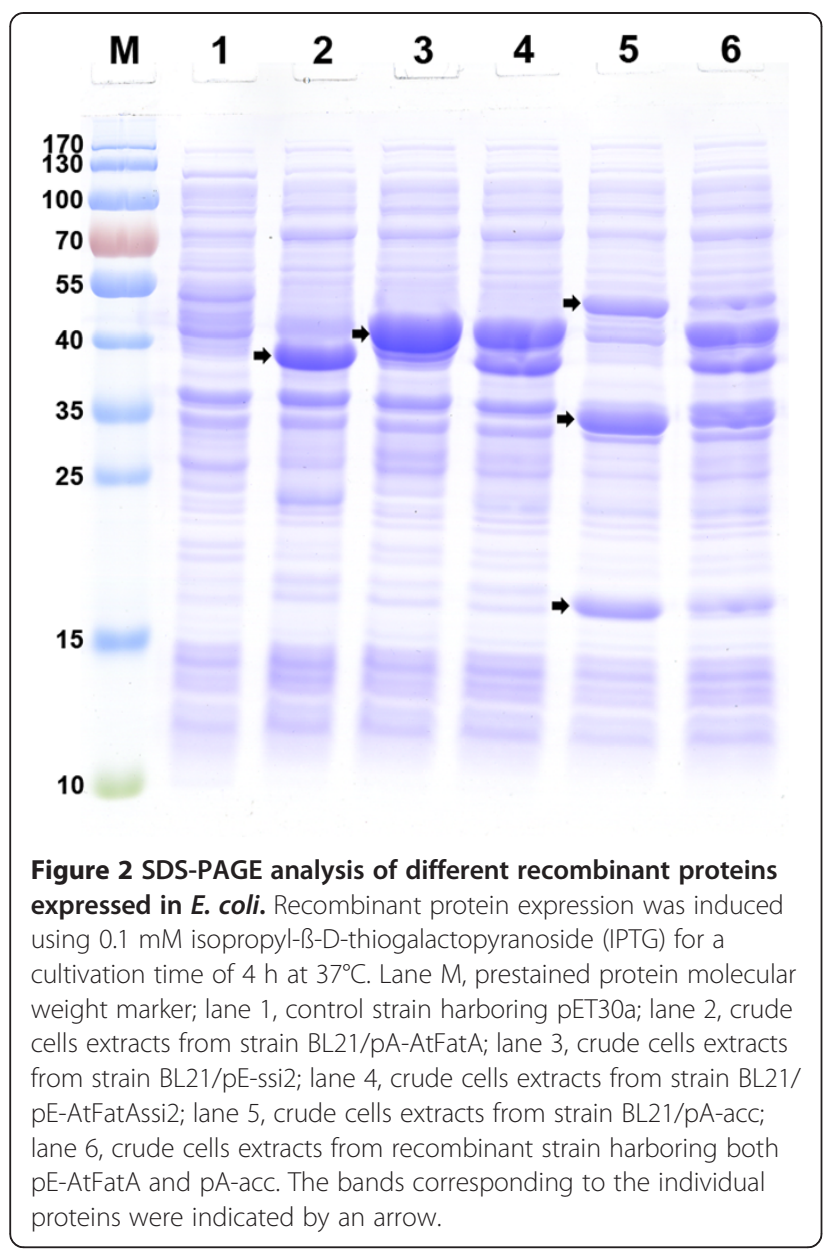

about $23.4 \mathrm{mg} / \mathrm{L}$ and cell density of the final culture broth reached an $\mathrm{OD}_{600}$ of 3.1. Although the palmitoyl-ACP pool was much larger in E. coli [39,40], palmitoleate levels of the FFA profiles in the strain heterologously expressing AtFatA were much greater than palmitate. This phenomenon can be interpreted by the fact that AtFatA preferred unsaturated acyl-ACP as its substrates rather than saturated acylACP. On the other hand, oleate $(18: 1 \Delta 9)$ is not the major constituent of $E$. coli fatty acids and cis-vaccenate seems not to be a good substrate for this enzyme. Thus, expression of AtFatA individually does not obviously increase the cis-vaccenate level. Compared with previous studies using thioesterases to produce FFAs in E. coli [41-43], the contents of free MUFAs were greatly enhanced. However, the total FFA production was much lower. The low FFA titer achieved in this study might be due to the less active AtFatA. The acyl-CoA thioesterases of bacterial origin seemed to be more effective for FFA production.

\section{Increasing unsaturated fatty-acid content by a fatty-acid desaturase}

The UFA biosynthesis pathway of $E$. coli is different from many other bacteria and higher plants. Wild-type
E. coli does not encode any fatty-acid desaturase. It employs an anaerobic pathway to synthesize UFAs. Fatty-acid desaturases are enzymes that introduce double bonds into the hydrocarbon chains of fatty acids. They are important to the maintenance of the proper structure and function of biological membranes [44]. Plant fatty-acid desaturases can use acyl chains attached to E. coli ACP as substrates and several of these enzymes have been characterized by previous researchers [45-47]. The fatty-acid desaturase SSI2 from Arabidopsis catalyzes the conversion of stearoyl (18:0)-ACP to oleoyl-ACP through the eukaryotic pathway of plant lipid biosynthesis [48]. Thus, we coexpressed this enzyme with the thioesterase AtFatA in E. coli. Figure 2, lane 3 (corresponding to the band of molecular weight $45.6 \mathrm{kDa}$ ) and lane 4 shows the electrophoresis spectrums of strain BL21/pE-ssi2 and BL21/pE-AtFatAssi2. Both of the two enzymes were successfully expressed in E. coli.

In order to investigate the effects of SSI2 on FFA compositions, recombinant $E$. coli strains harboring $\mathrm{pE}$ AtFatAssi2 were cultured under the same conditions for $16 \mathrm{~h}$ as discussed above. Samples were taken from the culture broth and fatty acids were analyzed by GC. The fatty-acid profiles of strain BL21/pA-AtFatA and BL21/ pE-AtFatAssi2 were compared in Table 2. We found that the introduction of the SSI2 desaturase did not affect total FFA production of the recombinant strain. However, the contents of the two UFAs increased when compared with the AtFatA solely expressed strain. The ratio of UFA to SFA reached about 3.4 in this engineered strain. As the optimal substrate stearoyl-ACP for the SSI2 desaturase was not present as the major component in $E$. coli, this enzyme used palmitoyl-ACP as an alternative substrate to produce palmitoleate. The overproduced palmitoleate was further elongated by $f a b F$ (encoding E. coli $\beta$-ketoacyl-ACP synthase II) [49], resulting in an enhanced cis-vaccenate level. Therefore, the contents of both of the two UFAs were improved to some extent.

\section{Inactivation of the native fatty-acid catabolic pathway}

E. coli can utilize long-chain fatty acids as its sole carbon and energy source, which might inhibit FFA production. Along with the development of modern molecular biology, the fatty-acid uptake mechanism has been largely resolved. E. coli has evolved a highly regulated fatty-acid transport system comprised of the outer membrane protein FadL, the periplasmic protein Tsp and the inner membrane-associated enzyme FadD [50]. Exogenous fatty acids could bind to the FadL protein with high affinity. With the help of the Tsp protein, fatty acids are transferred across the double membrane and finally activated by acyl-CoA synthetase, which is encoded by fadD [51]. Among the three proteins, FadD is shown to catalyze the rate-limiting step for fatty-acid utilization. 

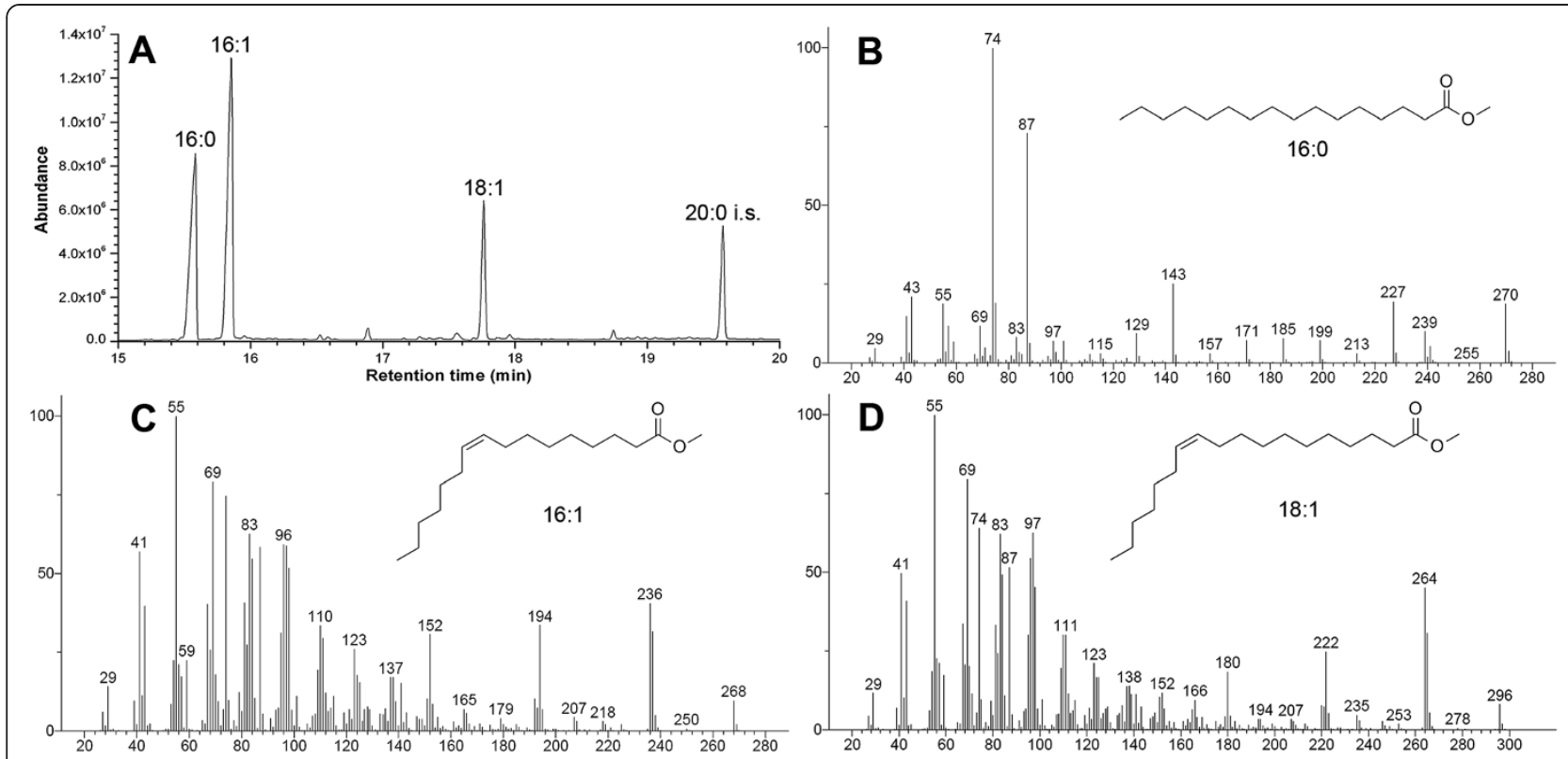

Figure 3 Identification of free fatty-acid compositions of the AtFatA-expressing strain by gas chromatography-mass spectrometry (GC-MS). (A) A total ion chromatogram (TIC) of free fatty-acid methyl esters from E. coli BL21 star(DE3) harboring pA-AtFatA after being induced for 16 h. (B) Mass spectrum of palmitate methyl ester (16:0); (C) mass spectrum of palmitoleate methyl ester (16:1 $\Delta 9$ ); (D) mass spectrum of cis-vaccenate methyl ester (18:1 $\Delta 11)$. The structures were matched by searching a standard NIST library. The molecular ion peaks of these fatty-acid methyl esters are marked with circles.

E. coli fadD mutants could accumulate FFAs released from membrane lipids in the stationary phase [52]. To further increase the total FFA content, we disrupted the fadD gene in the E. coli BL21 star(DE3) chromosome using the Red recombination method, resulting strain BL21 $\triangle$ fadD. Successful gene disruptions were confirmed by PCR amplification (see Additional file 1: Figure S1). Liquid growth tests on M9 minimal medium supplemented with palmitate as the sole carbon source further proved that the fatty-acid catabolism-blocked strain, BL21 $\triangle$ fadD, grew much more poorly than its parent strain.

With the aim to evaluate the effects of $f a d D$ disruption on free UFA production, the recombinant plasmid $\mathrm{pE}$ AtFatAssi2 was transformed into strain BL21 $\Delta$ fadD. The resulting strain $\mathrm{BL} 21 \Delta \mathrm{fadD} / \mathrm{pE}$-AtFatAssi2 was cultured under shake-flask conditions. Cell density and different types of FFA production were monitored over the course of the fermentation. As shown in Figure 4, the total FFA production was greatly enhanced in the fadD mutant strain. After being induced for $16 \mathrm{~h}$, the fadD mutant strain harboring pE-AtFatAssi2 accumulated $57.1 \mathrm{mg} / \mathrm{L}$ of FFAs, which was 2.4-fold that of strain BL21/pE-AtFatAssi2, and the cell growth was comparable between the two strains at this time point. Compared with previous reports $[14,20]$, disruption of the $f a d D$ gene showed similar effects on FFA production. Also, the ratio of UFAs remained stable between the two strains. These results demonstrated that knockout of the acyl-CoA synthetase enhanced FFA production without affecting the fatty-acid compositions.

\section{Boosting fatty-acid production by overexpressing native E. coli acetyl-CoA carboxylase}

The above strains successfully accumulated considerable amounts of free MUFAs in the culture broth. However, this level of production was still far beyond industrial applications. In biological systems, malonyl-CoA is the direct precursor for fatty-acid biosynthesis. However, E. coli only maintains a very low level of malonyl-CoA for natural anabolism [53]. The insufficient supply of intracellular malonyl-CoA hampers high-level FFA production. Acetyl-

Table 2 Effects of the fatty-acid desaturase SSI2 on free fatty-acid compositions

\begin{tabular}{lcccccc}
\hline Strains & OD $_{\mathbf{6 0 0}}$ & \multicolumn{4}{c}{ Free fatty acids $(\mathbf{m g} / \mathrm{L})$} \\
\cline { 3 - 7 } & & $\mathbf{1 6 : 1}$ & $\mathbf{1 6 : 0}$ & $\mathbf{1 8 : 1}$ & Total & UFA:SFA \\
\hline BL21/pA-AtFatA & $3.27 \pm 0.40$ & $10.7 \pm 2.6$ & $7.7 \pm 1.5$ & $5.0 \pm 1.1$ & $23.4 \pm 4.6$ & $2.0: 1$ \\
BL21/pE-AtFatAssi2 & $2.95 \pm 0.31$ & $10.6 \pm 2.2$ & $5.0 \pm 0.9$ & $6.2 \pm 1.0$ & $21.8 \pm 3.4$ & $3.4: 1$ \\
\hline
\end{tabular}

OD, optical density; UFA, unsaturated fatty acids; SFA, saturated fatty acids. The values represent mean \pm SD. 


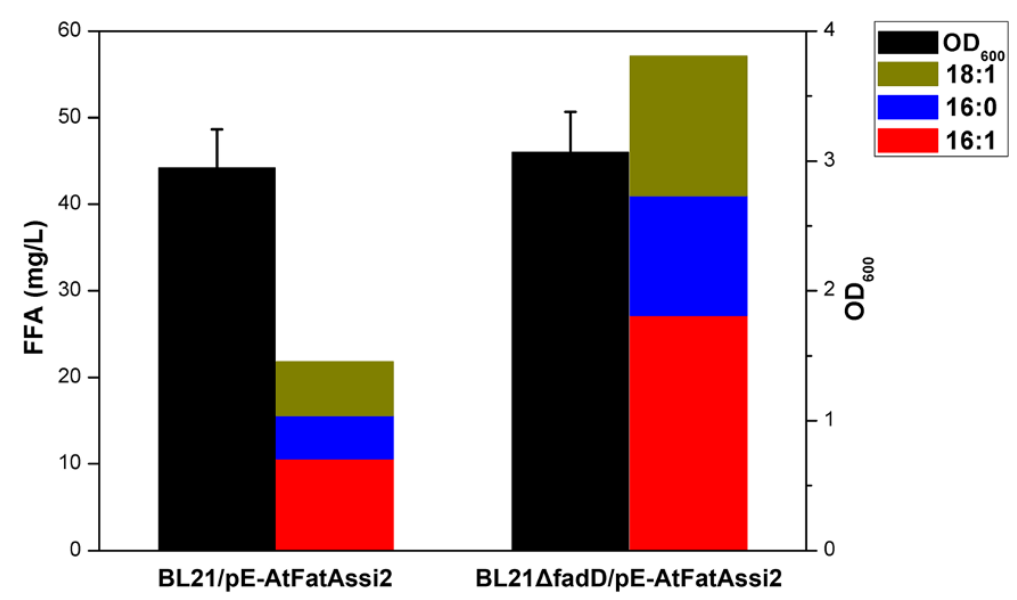

Figure 4 Effects of $\mathbf{f a d} \boldsymbol{D}$ knockout on free fatty-acid production. BL21/pE-AtFatAssi2, strain BL21 star(DE3) expressing Arabidopsis thioesterase AtFatA and fatty acid desaturase SSI2; BL21 $\triangle$ fadD/pE-AtFatAssi2, knockout of native fadD while expressing AtFatA and SSI2; 16:1, palmitoleate; 16:0, palmitate; 18:1, cis-vaccenate. Data were obtained after each strain was induced for $16 \mathrm{~h}$ in liquid M9 minimal medium supplemented with $1 \mathrm{mM} \mathrm{MgSO}_{4}$ and $20 \mathrm{~g} / \mathrm{L}$ glucose. FFA, free fatty acid; OD, optical density.

CoA carboxylase (ACCase), which catalyzes the irreversible carboxylation of acetyl-CoA, is the only producer of malonyl-CoA [54]. Previous studies have shown that overexpression of ACCase from different origins increased the pool of malonyl-CoA and the rate of fatty-acid synthesis $[55,56]$. Here, we overexpressed native $E$. coli ACCase together with AtFatA and ssi2 to further boost free MUFA production.

Figure 2, line 5 shows the gel electrophoresis pattern of the recombinant strain carrying pA-acc and line 6 is the recombinant strain carrying both pE-AtFatAssi2 and pA-acc. In all cases, the four subunits of ACCase were clearly expressed and we noted distinct bands of the expected size from bacterial extracts when compared with the control strain. Then strain BL21 $\Delta$ fadD/pE-AtFatAssi2\&pA-acc was cultured using M9 minimal medium under shake-flask conditions. FFA compositions of different engineered $E$. coli strains are presented in Table 3 and the corresponding GC chromatograms are provided (see Additional file 2: Figure S2). The amounts of FFA accumulated in the fermentation broth, the productivity of FFAs per cell dry weight (DW) and the yields of FFA on glucose of different recombinant strains were calculated and are shown in Figure 5. It could be seen that the ACCase overexpression strain produced more FFAs than other strains. The final titer of FFAs reached $82.6 \mathrm{mg} / \mathrm{L}$, which was 3.5 -fold that of the AtFatA solely expressed strain and 1.4-fold that of the fadD mutant strain. The productivity of FFAs per cell DW of the ACCase overexpression strain was $64.5 \mathrm{mg} / \mathrm{g}$ DW. The yield of FFAs on glucose of this strain also reached about $3.3 \%$ of the theoretical yield (the theoretical yield of $35.6 \%$ was formula):

$$
4 \text { Glucose } \rightarrow 8 \text { Acetyl-CoA } \rightarrow \text { Palmitate }
$$

This yield was far from the theoretical limits and much lower than many previous reports $[20,43,57]$. Considering the relatively low catalytic activity of the thioesterase AtFatA, we can expect to achieve a high titer of FFAs by improving the efficiency of this enzyme.

In addition, the recombinant strain $\mathrm{BL} 21 \Delta \mathrm{fadD} / \mathrm{pE}$ AtFatAssi2\&pA-acc accumulated FFAs much faster. FFA concentrations of the ACCase overexpression strain reached the maximum level after being induced for $12 \mathrm{~h}$, whereas the other strains required about $16 \mathrm{~h}$ to achieve the maximum titer. Fatty-acid composition analysis showed that overexpression of ACCase did not affect the

Table 3 Free fatty-acid compositions of different metabolically engineered $E$. coli strains

\begin{tabular}{|c|c|c|c|c|c|c|}
\hline \multirow[t]{2}{*}{ Strains } & \multicolumn{6}{|c|}{ Free fatty acids (\%) } \\
\hline & $14: 0$ & $16: 1$ & $16: 0$ & $17: 0 c^{*}$ & $18: 1$ & $19: 0 c^{* *}$ \\
\hline BL21/pA-AtFatA & 1.8 & 44.6 & 32.1 & 0.5 & 20.8 & 0.2 \\
\hline BL21/pE-AtFatAssi2 & 1.9 & 47.4 & 22.2 & 0.5 & 27.7 & 0.3 \\
\hline BL21 $\Delta \mathrm{fadD} / \mathrm{pE}-$ AtFatAssi2 & 1.5 & 47.4 & 22.8 & 0.2 & 27.9 & 0.2 \\
\hline BL21 $\triangle$ fadD/pE-AtFatAssi2\&pA-acc & 1.8 & 47.5 & 22.6 & 0.2 & 27.8 & 0.1 \\
\hline
\end{tabular}

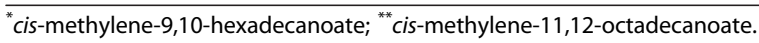




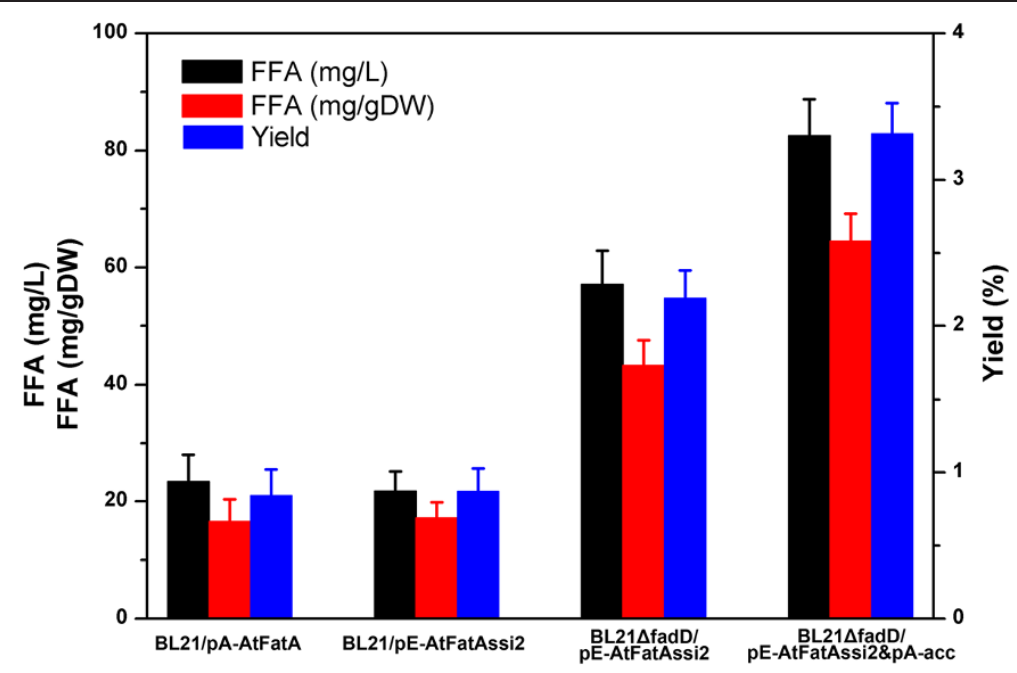

Figure 5 Comparison of free fatty-acid production and the yields on glucose of several different strains. BL21/pA-AtFatA, strain BL21 star (DE3) expressing Arabidopsis thioesterase; BL21/pE-AtFatAssi2, strain BL21 star(DE3) expressing Arabidopsis thioesterase and fatty-acid desaturase; BL21 $\triangle \mathrm{fadD} / \mathrm{pE}$-AtFatAssi2, knockout of native fadD while expressing AtFatA and SSI2; BL21 $\triangle \mathrm{fadD} / \mathrm{pE}$-AtFatAssi2\&pA-acc, further overexpression native of $E$. coli acetyl-CoA carboxylase. Data were obtained after each strain was induced for $16 \mathrm{~h}$ in liquid M9 minimal medium supplemented with $1 \mathrm{mM} \mathrm{MgSO}_{4}$ and $20 \mathrm{~g} / \mathrm{L}$ glucose except for the recombinant strain BL21 $\triangle$ fadD/pE-AtFatAssi2\&pA-acc (12 h). FFA, free fatty acid.

FFA constituents either. MUFAs were the predominant components of the total FFA profiles and the ratio of UFA to SFA was above 3:1.

\section{Fed-batch fermentation}

To further investigate the feasibility for larger-scale production of free MUFAs, the finally engineered strain
BL21 $\Delta$ fadD/pE-AtFatAssi2\&pA-acc was cultured in a 5L-scale laboratory fermenter. Cell density, residual glucose, and FFA production were monitored over the course of the experiment. The residual glucose was maintained at a very low level (below $1 \mathrm{~g} / \mathrm{L}$ ) throughout the whole fermentation process. Figure 6 shows the time profiles for cell density, and concentrations of different

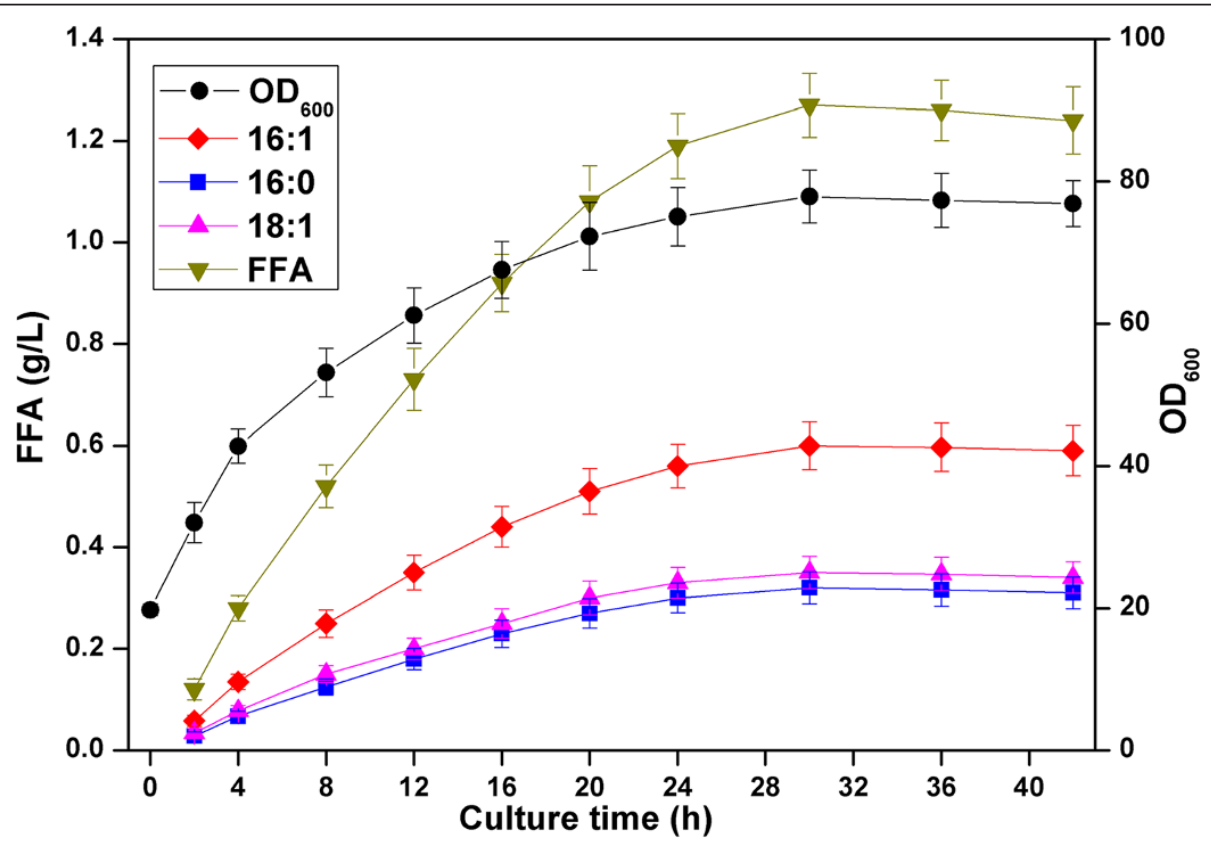

Figure 6 Time profiles for cell density $\left(\mathrm{OD}_{600}\right)$, palmitoleate $(16: 1 \Delta 9)$, palmitate $(16: 0)$ and cis-vaccenate $(18: 1 \Delta 11)$ and total free fatty-acid production during fed-batch fermentation of the finally engineered strain BL21 1 fadD/pE-AtFatAssi2\&pA-acc. FFA, free fatty acids; OD, optical density. 
types of FFA for 42-h fed-batch fermentations. For approximately $30 \mathrm{~h}$ post-induction, the bacteria grew very fast to an $\mathrm{OD}_{600}$ of 80 or so. FFAs accumulated rapidly in the culture broth. The highest FFA production was obtained after being induced for $30 \mathrm{~h}$, that is, $1.27 \mathrm{~g} / \mathrm{L}$, corresponding to a volumetric productivity of $5.4 \mathrm{mg} /$ $\left(\mathrm{L} \cdot \mathrm{h} \cdot \mathrm{OD}_{600}\right)$. The titers of palmitoleate, palmitate and cis-vaccenate increased gradually along with the bacterial cell growth, and the ratios of these three fatty acids remained stable. The two types of UFA, palmitoleate and cis-vaccenate, made up of more than $75 \%$ of the total FFA profiles. The above results obtained under the fermentor level demonstrated that this engineered E. coli strain had the potential to produce free MUFAs on a large scale. Compared with many other fatty-acid-producing systems [42,57], the final titer of FFAs was not very high and its industrial application was still far from occurring. However, the fatty-acid composition was greatly improved as SFAs always made up more than $60 \%$ of the total fattyacid profiles in previous strains $[20,42,57]$.

\section{Conclusions}

In this study, we have successfully constructed an engineered E. coli strain capable of producing high levels of free MUFAs. By introducing four distinct genetic variations targeted at fatty-acid production and saturationlevel regulation, the finally engineered strain produced $1.27 \mathrm{~g} / \mathrm{L}$ of FFAs and the major proportions of these FFAs were MUFAs (palmitoleate and cis-vaccenate). Considering both the excellent oxidative stability and cold-flow properties of biodiesel derived from MUFAs, the fuel manufactured by the current $E$. coli strain would be more suitable for diesel engines. In addition, the results of this work would give some implications to improve the quality of biodiesel from higher plants and microalgae.

\section{Methods}

\section{Bacterial strains}

$\Delta \mathrm{A}$ list of bacterial strains and recombinant plasmids used in this study is presented in Table 4. The onestep gene inactivation strategy, previously described by Datsenko and Wanner [58], was applied to knock out the chromosomal genes in E. coli BL21 star(DE3). Oligonucleotide primers used for gene disruption are listed (see Additional file 3: Table S1). For the construction of strain BL21 $\triangle \mathrm{fadD}$, a linear DNA fragment containing the FRT-flanked kanamycin resistance cassette was amplified with primers fadD_Del_F and fadD_Del_R from plasmid $\mathrm{pKD} 4$. The obtained disrupting fragments were electrotransformed into E. coli competent cells that carried the Red recombinase expression vector pKD46 and integrated into its chromosome. Successfully disrupted colonies were then transformed with plasmid pCP20 and induced at $42^{\circ} \mathrm{C}$ to eliminate the kanamycin resistance. PCR verifications were performed using primer pairs designed according to the sequences up- and downstream of disrupted regions (fadD_DelIden_F and fadD_DelIden_R).

\section{Plasmids construction}

The fatty-acid desaturase from A. thaliana (ssi2) was cloned to the expression vector pET30a resulting plasmid pE-ssi2 in a previous study [59]. The four subunits of native $E$. coli acetyl-CoA carboxylase were also cloned into a single expression vector pACYCduet-1, resulting pA-acc (see Additional file 4: Figure S3) in another study

Table 4 Strains and plasmids used in this study

\begin{tabular}{|c|c|c|}
\hline Strains or plasmids & Genotype/description & Sources \\
\hline \multicolumn{3}{|l|}{ Strains } \\
\hline E. coli BL21 star(DE3) & $F^{-}$ompthsdS ${ }_{B}\left(r_{B}^{-} m_{B}^{-}\right)$gal dcm rne131 (DE3) & Invitrogen \\
\hline E. coli BL21 star(DE3) $\Delta \mathrm{fadD}$ & Knockout of fadD encoding acyl-CoA synthetase & This study \\
\hline \multicolumn{3}{|l|}{ Plasmids } \\
\hline pACYCduet-1 & $\mathrm{Cm}^{r}$ oriP15A lacl ${ }^{9} \mathrm{~T} 7 \mathrm{p}$ & Novagen \\
\hline pET30a & Kan $^{r}$ oripBR322 $\operatorname{lac}^{q}{ }^{T} T p$ & Novagen \\
\hline pEASY-Blunt & $\mathrm{Kan}^{r} \mathrm{Amp} \mathrm{p}^{r}$ oripUC & Transgen \\
\hline pE-ssi2 & pET30a harboring Arabidopsis fatty-acid desaturase & [59] \\
\hline pA-acc & pACYCduet-1 harboring E. coli acetyl-CoA carboxylase & {$[60]$} \\
\hline PEASY-AtFatA & pEASY-Blunt harboring Arabidopsis thioesterase & This study \\
\hline pA-AtFatA & pACYCduet-1 harboring Arabidopsis thioesterase & This study \\
\hline pE-AtFatAssi2 & pET30a harboring Arabidopsis fatty-acid desaturase and thioesterase & This study \\
\hline pKD46 & $A p^{r} \operatorname{oriR} 101 \operatorname{rep} A(\mathrm{Ts}) \lambda \operatorname{Red}(\gamma, \beta$ and exo) & Coli Genetic Stock Center \\
\hline pKD4 & FRT-Kan'-FRT oriR6K & Coli Genetic Stock Center \\
\hline pCP20 & $A p^{r} C m^{r}$ repA(Ts) FLP & Coli Genetic Stock Center \\
\hline
\end{tabular}


[60]. The Arabidopsis fatty acyl-ACP thioesterase (AtFatA) [AK176105: GenBank] gene was amplified using cDNA of Arabidopsis as a template and with primers AtFatA_F_NcoI and AtFatA_R_Sall containing the start and stop codons as well as the restriction sites of NcoI and SalI. The amplified PCR products were analyzed by agarose gel electrophoresis and directly ligated into the pEASY-Blunt vector (Transgen, Beijing, China), resulting pEASYAtFatA. The ligation products were transformed into $E$. coli DH5a-competent cells by heat-pulse transformation, and the antibiotic resistant transformants were selected to sequence AtFatA. The plasmid pEASY-AtFatA was doubledigested with $\mathrm{NcoI}$ and SalI, and the digested AtFatA fragment was withdrawn and ligated to pACYCduet-1 expression vector predigested with the same restriction enzymes and generated pA-AtFatA. PCR reaction was performed using $\mathrm{pA}-\mathrm{AtF}$ atA as a template and a primer pair that allowed the amplification of the T7 promoter sequence along with the AtFatA structural gene. The PCR product, T7AtFatA was then cloned into $\mathrm{pE}$-ssi2 between SalI and NotI sites, to create pE-AtFatAssi2 (see Additional file 5: Figure S4). Successful gene cloning was verified by colony $\mathrm{PCR}$, restriction mapping and direct nucleotide sequencing.

\section{Protein expression and gel electrophoresis analysis}

Single colonies of $E$. coli strains harboring different recombinant plasmids were used to inoculate liquid LB medium containing appropriate antibiotics and grown overnight at $37^{\circ} \mathrm{C}$. The saturated culture was diluted 1:100 in fresh LB medium and incubated under the same conditions. When the $\mathrm{OD}_{600}$ reached about 0.6, IPTG was added to a final concentration of $0.1 \mathrm{mM}$, and cell growth was continued for $4 \mathrm{~h}$. The cells pelleted from $5 \mathrm{ml}$ of culture were suspended in Tris- $\mathrm{HCl}$ buffer $(\mathrm{pH} 8.0)$ and subjected to ultrasonication. The mixture was centrifuged and the supernatant obtained was mixed with $2 \times$ sodium dodecyl sulfate (SDS) sample buffer, heated at $100^{\circ} \mathrm{C}$ for 10 minutes and then analyzed by SDS-PAGE.

\section{Shake-flask level cultivation}

Shake-flask experiments were carried out in triplicate series of 250-ml Erlenmeyer flasks containing $50 \mathrm{ml}$ of M9 minimal medium $\left(6 \mathrm{~g} / \mathrm{L} \mathrm{Na}_{2} \mathrm{HPO}_{4}, 3 \mathrm{~g} / \mathrm{L} \mathrm{KH}_{2} \mathrm{PO}_{4}\right.$, $1 \mathrm{~g} / \mathrm{L} \mathrm{NH}_{4} \mathrm{Cl}$ and $0.5 \mathrm{~g} / \mathrm{L} \mathrm{NaCl}$ ) supplemented with $1 \mathrm{mM} \mathrm{MgSO}_{4}$ and $20 \mathrm{~g} / \mathrm{L}$ glucose as the carbon source. E. coli strains harboring different recombinant plasmids were inoculated to the culture medium and incubated in a gyratory shaker incubator at $37^{\circ} \mathrm{C}$ and $200 \mathrm{rpm}$. When the $\mathrm{OD}_{600}$ of the culture reached about 0.6. IPTG was added to a final concentration of $0.1 \mathrm{mM}$ to induce recombinant protein expression and FFA production. The culture temperature was then switched to $30^{\circ} \mathrm{C}$. Cell density, residual glucose and FFA production were measured during the whole fermentation courses.

\section{Fermentor scale cultivation}

For large-scale production of free MUFAs, fed-batch cultivation was carried out in a Biostat B plus MO5L fermentor (Sartorius, Göttingen, Germany) containing $3 \mathrm{~L}$ of growth medium $\left(3 \mathrm{~g} / \mathrm{L}\left(\mathrm{NH}_{4}\right)_{2} \mathrm{SO}_{4}, 1 \mathrm{~g} / \mathrm{L}\right.$ citrate,

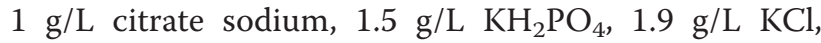
$75.6 \mathrm{mg} / \mathrm{L} \mathrm{FeSO}_{4}$ ) that was sterilized at $115^{\circ} \mathrm{C}$ for $30 \mathrm{mi}-$ nutes. Glucose $(20 \mathrm{~g} / \mathrm{L}), \mathrm{MgSO}_{4}(3 \mathrm{~g} / \mathrm{L})$ and trace elements $\left(1 \mathrm{ml} / \mathrm{L}, 3.7 \mathrm{~g} / \mathrm{L}\left(\mathrm{NH}_{4}\right)_{6} \mathrm{Mo}_{7} \mathrm{O}_{24} \cdot 4 \mathrm{H}_{2} \mathrm{O}, 2.9 \mathrm{~g} / \mathrm{L}\right.$ $\mathrm{ZnSO}_{4} \cdot 7 \mathrm{H}_{2} \mathrm{O}, 24.7 \mathrm{~g} / \mathrm{L} \mathrm{H}_{3} \mathrm{BO}_{3}, 2.5 \mathrm{~g} / \mathrm{L} \mathrm{CuSO}_{4} \cdot 5 \mathrm{H}_{2} \mathrm{O}$, $15.8 \mathrm{~g} / \mathrm{L} \mathrm{MnCl}_{2} \cdot 4 \mathrm{H}_{2} \mathrm{O}$ ) were autoclaved or filtersterilized separately and added prior to initiation of the fermentation. Then, $50 \mathrm{ml}$ of inoculum was prepared by incubating the culture in shake flasks containing liquid LB medium overnight at $37^{\circ} \mathrm{C}$. The fermentation was first operated in a batch mode and the control settings were: $37^{\circ} \mathrm{C}$, stirring speed $600 \mathrm{rpm}$, and airflow $2 \mathrm{~L} /$ minute. During the fermentation process, the $\mathrm{pH}$ was controlled at 7.0 via automated addition of ammonia. Antifoam 204 was added to prohibit foam development. The agitation was associated with the dissolved oxygen (DO) to maintain a DO concentration above $20 \%$ saturation. After the initial glucose was nearly exhausted, fed-batch mode was commenced by feeding a solution containing $50 \%$ of glucose at appropriate rates and the residual glucose was maintained at a very low level. When the cells were grown to an $\mathrm{OD}_{600}$ of about 20, IPTG was added to the culture at a concentration of $0.5 \mathrm{mM}$ and the culture temperature was switched to $30^{\circ} \mathrm{C}$. Samples of fermentation broth were taken at appropriate intervals to determine cell density and FFA production.

\section{Free fatty-acid extraction and fatty-acid methyl esters preparation}

FFAs were extracted from the culture broth following the procedure of Steen et al. [14] with some modifications. $0.4 \mathrm{ml}$ of $\mathrm{HCl}$ and $4 \mathrm{ml}$ of ethyl acetate were added to $4 \mathrm{ml}$ of culture broth, spiked with $10 \mathrm{mg} / \mathrm{L}$ of arachidate as an internal standard. The mixture was vortexed for $30 \mathrm{~s}$ followed by shaking at $200 \mathrm{rpm}$ for $30 \mathrm{mi}-$ nutes. The organic layer was separated and a second extraction was performed by the addition of another $4 \mathrm{ml}$ of ethyl acetate.

Fatty acid methyl esters (FAMEs) were prepared according a standard procedure [61]. FFAs extracted in the ethyl acetate phase were evaporated to dryness under a stream of nitrogen, then suspended in $3 \mathrm{ml}$ of boron trifluoride/methanol ( $1: 4$, by volume) and heated at $70^{\circ} \mathrm{C}$ for 30 minutes in sealed tubes. Esterified fatty acids were extracted by addition of $3 \mathrm{ml}$ of hexane. 


\section{Analytical methods}

Cell density was determined by measuring the absorbance of the culture broth at $600 \mathrm{~nm}$ (an $\mathrm{OD}_{600}$ of 1.0 corresponds to $0.43 \mathrm{~g}$ dry cell weight per liter). The residual glucose in the culture medium was quantified using an SBA-40D Biological Sensing Analyzer (Biology Institute of Shandong Academy of Sciences, China).

FAMEs were identified using an Agilent (Santa Clara, United States) 7890A GC coupled to an Agilent 5975C quadrupole mass detector. The GC-MS conditions were as follows: a 30-m HP-INNOWax column (internal diameter $0.25 \mathrm{~mm}$, film thickness $0.25 \mu \mathrm{m}$ ); an oven temperature program composed of an initial hold at $100^{\circ} \mathrm{C}$ for 5 minutes, ramping at $10^{\circ} \mathrm{C}$ per minute to $250^{\circ} \mathrm{C}$, and a final hold at $250^{\circ} \mathrm{C}$ for 3 minutes; highpurity nitrogen as carrier gas with a linear velocity of $1 \mathrm{ml} /$ minute; an ion source temperature of $220^{\circ} \mathrm{C}$ and $\mathrm{EI}$ ionization at $70 \mathrm{eV}$.

FAMEs contents were determined by a Varian (Palo Alto, United States) GC-450 system equipped with a $30 \mathrm{~m}$ HP-5 column (internal diameter $0.32 \mathrm{~mm}$, film thickness $0.25 \mu \mathrm{m}$ ) and using high-purity nitrogen as carrier gas with a linear velocity of $1 \mathrm{ml} /$ minute. The injector temperature was $250^{\circ} \mathrm{C}$, the FID temperature was $300^{\circ} \mathrm{C}$ and the split ratio was $1: 10$. The oven was initially set at $100^{\circ} \mathrm{C}$ for 5 minutes, increased at $20^{\circ} \mathrm{C}$ per minute to $160^{\circ} \mathrm{C}$, then ramped at $10^{\circ} \mathrm{C}$ per minute to $250^{\circ} \mathrm{C}$ and finally held at $250^{\circ} \mathrm{C}$ for 3 minutes.

\section{Additional files}

Additional file 1: Figure S1. Identification of the fadD knockout E. coli strains. PCR verifications were performed with primers fadD_Dellden_F and fadD_Dellden_R (Additional file 3: Table S1) corresponding to sequences up- and downstream of the disrupted regions. Lane M, DNA molecular weight markers; lane 1, strain BL21/ $\Delta$ fadD by disrupting the fadD gene; lane 2 the original strain BL21 star(DE3).

Additional file 2: Figure S2. GC chromatogram of free fatty-acid methyl esters from different metabolically engineered $E$. coli strains. A, BL21/pA-AtFatA; B, BL21/pE-AtFatAssi2; C, BL21 1 fadD/pE-AtFatAssi2; D, BL21 $\Delta \mathrm{fadD} / \mathrm{pE}-\mathrm{AtF} a \mathrm{tAssi2} \& \mathrm{pA}$-acc.

Additional file 3: Table S1. Primers used in this study for gene disruption, verification and plasmids construction.

Additional file 4: Figure S3. The recombinant plasmid pA-acc overexpressing the four subunits of native E. coli acetyl-CoA carboxylase.

Additional file 5: Figure S4. The recombinant plasmid pE-AtFatAssi2 coexpressing the acyl-ACP thioesterase AtFatA and the fatty-acid desaturase SSI2.

\section{Abbreviations}

ACCase: Acetyl-CoA carboxylase; ACP: acyl carrier protein; AtFatA: gene encoding a FatA-type thioesterase from Arabidopsis; DO: dissolved oxygen; DW: dry weight; FAME: fatty-acid methyl esters; FAS: fatty acid synthase; FFA: free fatty acid: FID: flame ionization detector; GC-MS: gas chromatography-mass spectrometry; IPTG: isopropyl-ß-D-thiogalactopyranoside; MUFA: monounsaturated fatty acid; OD: optical density; PFUA: polyunsaturated fatty acid; SFA: saturated fatty acid; ssi2: gene encoding a fatty-acid desaturase from Arabidopsis; UFA: unsaturated fatty acid.

\section{Competing interests}

The authors declare that they have no competing interests.

\section{Authors' contributions}

YC: conception and design, data collection and analysis, manuscript writing and final approval of the manuscript. WL: data collection and analysis, critical revision and final approval of the manuscript. XX: data collection, critical revision and final approval of the manuscript. $\mathrm{HZ}$ : data collection, critical revision and final approval of the manuscript. JW: data analysis, critical revision and final approval of the manuscript. MX: conception and design, financial support, manuscript writing and final approval of manuscript. All authors read and approved the final manuscript.

\section{Acknowledgements}

This work was sponsored by the National Natural Science Foundation of China (No. 21202179, 21376255 and 31200030), Main Direction Program of Knowledge Innovation of CAS (KSCX2-EW-G-13), Key Technologies Research and Development Program of China (No. 2012BAD32B06), Sci-Tech Development Project of Qingdao (No. 12-1-4-9-(3)-jch). The authors would like to thank Dr Haiyan Yang and Yun Fa for GC analysis and Dr Cong Wang, Wenna Guan and Fali Bai for GC-MS analysis of fatty-acid methyl esters.

Received: 18 August 2013 Accepted: 17 March 2014 Published: 10 April 2014

\section{References}

1. Knothe G: Dependence of biodiesel fuel properties on the structure of fatty acid alkyl esters. Fuel Process Technol 2005, 86:1059-1070.

2. Knothe G: "Designer" biodiesel: optimizing fatty ester composition to improve fuel properties. Energy Fuels 2008, 22:1358-1364.

3. Knothe G, Steidley KR: Kinematic viscosity of fatty acid methyl esters: prediction, calculated viscosity contribution of esters with unavailable data, and carbon-oxygen equivalents. Fuel 2011, 90:3217-3224.

4. Laboratory NRE: Biodiesel Handling and Use Guidelines. 3rd edition. Oak Ridge; 2006

5. Qu J, Mao HZ, Chen W, Gao SQ, Bai YN, Sun YW, Geng YF, Ye J: Development of marker-free transgenic Jatropha plants with increased levels of seed oleic acid. Biotechnol Biofuels 2012, 5:10.

6. Puhan S, Saravanan N, Nagarajan G, Vedaraman N: Effect of biodiesel unsaturated fatty acid on combustion characteristics of a DI compression ignition engine. Biomass Bioenerg 2010, 34:1079-1088.

7. Knothe G: Fuel properties of highly polyunsaturated fatty acid methyl esters: prediction of fuel properties of algal biodiesel. Energy Fuels 2012, 26:5265-5273.

8. Cao Y, Yang J, Xian M, Xu X, Liu W: Increasing unsaturated fatty acid contents in Escherichia coli by coexpression of three different genes. Appl Microbiol Biotechnol 2010, 87:271-280.

9. Chisti Y: Biodiesel from microalgae. Biotechnol Adv 2007, 25:294-306.

10. Saenge C, Cheirsilp B, Suksaroge TT, Bourtoom T: Potential use of oleaginous red yeast Rhodotorula glutinis for the bioconversion of crude glycerol from biodiesel plant to lipids and carotenoids. Process Biochem 2011, 46:210-218.

11. Vicente G, Bautista LF, Rodríguez R, Gutiérrez FJ, Sádaba I, Ruiz-Vázquez RM, Torres-Martínez S, Garre V: Biodiesel production from biomass of an oleaginous fungus. Biochem Eng J 2009, 48:22-27.

12. Cao Y, Cao Y, Zhao MA: Biotechnological production of eicosapentaenoic acid: from a metabolic engineering point of view. Process Biochem 2012, 47:1320-1326.

13. Cao $Y$, Xian M, Zou H, Zhang H: Metabolic engineering of Escherichia coli for the production of xylonate. PLOS ONE 2013, 8:e67305.

14. Steen EJ, Kang YS, Bokinsky G, Hu ZH, Schirmer A, McClure A, del Cardayre SB, Keasling JD: Microbial production of fatty-acid-derived fuels and chemicals from plant biomass. Nature 2010, 463:559-562.

15. Lennen RM, Kruziki MA, Kumar K, Zinkel RA, Burnum KE, Lipton MS, Hoover SW, Ranatunga DR, Wittkopp TM, Marner WD, Pfleger BF: Membrane stresses induced by overproduction of free fatty acids in Escherichia coli. Appl Environ Microbiol 2011, 77:8114-8128.

16. Kalscheuer R, Stölting T, Steinbüchel A: Microdiesel: Escherichia coli engineered for fuel production. Microbiology 2006, 152:2529-2536. 
17. Elbahloul $Y$, Steinbüchel A: Pilot-scale production of fatty acid ethyl esters by an engineered Escherichia coli strain harboring the $\mathrm{p}$ (Microdiesel) plasmid. Appl Environ Microbiol 2010, 76:4560-4565.

18. Lennen RM, Pfleger BF: Engineering Escherichia coli to synthesize free fatty acids. Trends Biotechnol 2012, 30:659-667.

19. Dellomonaco C, Clomburg JM, Miller EN, Gonzalez R: Engineered reversal of the $\beta$-oxidation cycle for the synthesis of fuels and chemicals. Nature 2011, 476:355-359.

20. Lu X, Vora H, Khosla C: Overproduction of free fatty acids in E. coli: implications for biodiesel production. Metab Eng 2008, 10:333-339.

21. Marr AG, Ingraham JL: Effect of temperature on the composition of fatty acids in Escherichia coli. J Bacteriol 1962, 84:1260-1267.

22. Rashid U, Anwar F, Moser BR, Knothe G: Moringa oleifera oil: a possible source of biodiesel. Bioresour Technol 2008, 99:8175-8179.

23. Bi Y, Ding D, Wang D: Low-melting-point biodiesel derived from corn oil via urea complexation. Bioresour Technol 2010, 101:1220-1226.

24. Rashid U, Anwar F: Production of biodiesel through optimized alkalinecatalyzed transesterification of rapeseed oil. Fuel 2008, 87:265-273.

25. Satyanarayana M, Muraleedharan C: Comparative studies of biodiesel production from rubber seed oil, coconut oil, and palm oil including thermogravimetric analysis. Energ Source Part A 2011, 33:925-937.

26. Okullo AA, Temu AK, Ogwok P, Ntalikwa JW: Physico-chemical properties of biodiesel from jatropha and castor oils. Int J Renew Energy Res 2012, 2:47-52.

27. Magnuson K, Jackowski S, Rock CO, Cronan JE: Regulation of fatty acid biosynthesis in Escherichia coli. Microbiol Rev 1993, 57:522-542.

28. Marrakchi $\mathrm{H}$, Zhang YM, Rock CO: Mechanistic diversity and regulation of type II fatty acid synthesis. Biochem Soc Trans 2002, 30:1050-1055.

29. Cho H, Cronan JE: Escherichia coli thioesterase I, molecular cloning and sequencing of the structural gene and identification as a periplasmic enzyme. J Biol Chem 1993, 268:9238-9245.

30. Spencer AK, Greenspan AD, Cronan JE: Thioesterases I and II of Escherichia coli: hydrolysis of native acyl-acyl carrier protein thioesters. J Biol Chem 1978, 253:5922-5926.

31. Jones A, Davies HM, Voelker TA: Palmitoyl-acyl carrier protein (ACP) thioesterase and the evolutionary origin of plant acyl-ACP thioesterases. Plant Cell 1995, 7:359-371.

32. Voelker TA, Davies HM: Alteration of the specificity and regulation of fatty acid synthesis of Escherichia coli by expression of a plant medium-chain acyl-acyl carrier protein thioesterase. J Bacteriol 1994, 176:7320-7327.

33. Huynh TT, Pirtle RM, Chapman KD: Expression of a Gossypium hirsutum cDNA encoding a FatB palmitoyl-acyl carrier protein thioesterase in Escherichia coli. Plant Physiol Biochem 2002, 40:1-9.

34. Jha JK, Maiti MK, Bhattacharjee A, Basu A, Sen PC, Sen SK: Cloning and functional expression of an acyl-ACP thioesterase FatB type from Diploknema (Madhuca) butyracea seeds in Escherichia coli. Plant Physiol Biochem 2006, 44:645-655.

35. Bonaventure G, Bao X, Ohlrogge J, Pollard M: Metabolic responses to the reduction in palmitate caused by disruption of the FATB gene in Arabidopsis. Plant Physiol 2004, 135:1269-1279.

36. Salas JJ, Ohlrogge JB: Characterization of substrate specificity of plant FatA and FatB acyl-ACP thioesterases. Arch Biochem Biophys 2002, 403:25-34.

37. Chang YY, Cronan JE: Membrane cyclopropane fatty acid content is a major factor in acid resistance of Escherichia coli. Mol Microbiol 1999, 33:249-259.

38. Wang AY, Grogan DW, Cronan JE: Cyclopropane fatty acid synthase of Escherichia coli: Deduced amino acid sequence, purification, and studies of the enzyme active site. Biochemistry 1992, 31:11020-11028.

39. Ohlrogge J, Savage L, Jaworski J, Voelker T, Post-Beittenmiller D: Alteration of acyl-acyl carrier protein pools and acetyl-CoA carboxylase expression in Escherichia coli by a plant medium-chain acyl-acyl carrier protein thioesterase. Arch Biochem Biophys 1995, 317:185-190.

40. Rock CO, Jackowski S: Regulation of phospholipid synthesis in Escherichia coli: composition of the acyl-acyl carrier protein pool in vivo. J Biol Chem 1982, 257:10759-10765.

41. Li M, Zhang X, Agrawal A, San K-Y: Effect of acetate formation pathway and long chain fatty acid CoA-ligase on the free fatty acid production in E. coli expressing acy-ACP thioesterase from Ricinus communis. Metab Eng 2012, 14:380-387.
42. Zhang $X$, Li M, Agrawal A, San KY: Efficient free fatty acid production in Escherichia coli using plant acyl-ACP thioesterases. Metab Eng 2011, 13:713-722

43. Zheng Y, Li L, Liu Q, Qin W, Yang J, Cao Y, Jiang X, Zhao G, Xian M: Boosting the free fatty acid synthesis of Escherichia coli by expression of a cytosolic Acinetobacter baylyi thioesterase. Biotechnol Biofuels 2012, 5:76.

44. Los DA, Murata N: Structure and expression of fatty acid desaturases. BBA-Lipid Lipid Met 1998, 1394:3-15.

45. Shanklin J, Somerville C: Stearoyl-acyl-carrier-protein desaturase from higher plants is structurally unrelated to the animal and fungal homologs. Proc Natl Acad Sci USA 1991, 88:2510-2514.

46. Gummeson PO, Lenman M, Lee M, Singh S, Stymne S: Characterisation of acyl-ACP desaturases from Macadamia integrifolia Maiden \& Betche and Nerium oleander L. Plant Sci 2000, 154:53-60.

47. Whittle E, Cahoon EB, Subrahmanyam S, Shanklin J: A multifunctional acylacyl carrier protein desaturase from Hedera helix L. (English Ivy) can synthesize 16-and 18-carbon monoene and diene products. J Biol Chem 2005, 280:28169-28176.

48. Kachroo P, Shanklin J, Shah J, Whittle EJ, Klessig DF: A fatty acid desaturase modulates the activation of defense signaling pathways in plants. Proc Natl Acad Sci USA 2001, 98:9448-9453.

49. Edwards P, Sabo Nelsen J, Metz JG, Dehesh K: Cloning of the fabF gene in an expression vector and in vitro characterization of recombinant fabF and fabB encoded enzymes from Escherichia coli. FEBS Lett 1997, 402:62-66.

50. DiRusso CC, Black PN: Bacterial long chain fatty acid transport: gateway to a fatty acid-responsive signaling system. J Biol Chem 2004, 279:49563-49566.

51. Yoo JH, Cheng OH, Gerber GE: Determination of the native form of FadD, the Escherichia coli fatty acyl-CoA synthetase, and characterization of limited proteolysis by outer membrane protease OmpT. Biochem J 2001, 360:699-706.

52. Pech-Canul Á, Nogales J, Miranda-Molina A, Álvarez L, Geiger O, Soto MJ, López-Lara IM: FadD is required for utilization of endogenous fatty acids released from membrane lipids. J Bacteriol 2011, 193:6295-6304.

53. Zha W, Rubin-Pitel SB, Shao Z, Zhao H: Improving cellular malonyl-CoA level in Escherichia coli via metabolic engineering. Metab Eng 2009, 11:192-198.

54. Cronan JE, Waldrop GL: Multi-subunit acetyl-CoA carboxylases. Prog Lipid Res 2002, 41:407-435.

55. Davis MS, Solbiati J, Cronan JE: Overproduction of acetyl-CoA carboxylase activity increases the rate of fatty acid biosynthesis in Escherichia coli. J Biol Chem 2000, 275:28593-28598.

56. Lennen RM, Braden DJ, West RM, Dumesic JA, Pfleger BF: A process for microbial hydrocarbon synthesis: overproduction of fatty acids in Escherichia coli and catalytic conversion to alkanes. Biotechnol Bioeng 2010, 106:193-202

57. Liu T, Vora H, Khosla C: Quantitative analysis and engineering of fatty acid biosynthesis in E. coli. Metab Eng 2010, 12:378-386.

58. Datsenko KA, Wanner BL: One-step inactivation of chromosomal genes in Escherichia coli K-12 using PCR products. Proc Natl Acad Sci USA 2000, 97:6640-6645

59. Cao $Y$, Xian M, Yang J, Xu X, Liu W, Li L: Heterologous expression of stearoyl-acyl carrier protein desaturase (S-ACP-DES) from Arabidopsis thaliana in Escherichia coli. Protein Expr Purif 2010, 69:209-214.

60. Cao YJ, Jiang XL, Zhang RB, Xian M: Improved phloroglucinol production by metabolically engineered Escherichia coli. Appl Microbiol Biotechnol 2011, 91:1545-1552.

61. Bligh EG, Dyer WJ: A rapid method of total lipid extraction and purification. Can J Biochem Phys 1959, 37:911-917.

doi:10.1186/1754-6834-7-59

Cite this article as: Cao et al:: Production of free monounsaturated fatty acids by metabolically engineered Escherichia coli. Biotechnology for Biofuels 2014 7:59. 